UNIVERSIDAD NACIONAL DE LA PLATA • FACULTAD DE ODONTOLOGÍA

TESIS DE MAESTRÍA

\title{
TEMA: ANÁLISIS DE EDUCACIÓN PERMANENTE EN SALUD ODONTOLÓGICA Y EN EL ÁREA DE LA PREVENCIÓN
}

Autor: Dra. Leticia Argentina Rueda

Directora de Tesis: Prof. Dra. María Mercedes Medina

Año: 2012 


\section{INTRODUCCIÓN}




\section{INTRODUCCIÓN}

Las razones por las cuales se decide formar un determinado tipo de profesional no son generalmente bien definidas.

Cuando se mencionan algunas cuestiones, éstas difieren bastante de la práctica; porque se aspira a formar un profesional que pueda contar con las herramientas básicas que le posibiliten el desempeño de su profesión bajo diferente situaciones.

Cuando la evaluación de los planes de estudio abordan distintos aspectos que hacen a la profesión, aparecen diferencias de interpretación en los profesionales educadores.

$\mathrm{Ha}$ sido de nuestro interés, en nuestra investigación indagar de qué manera el proceso de enseñanza-aprendizaje está efectuándose.

Si resulta apropiado para la formación del Odontólogo. Si lo habilita a ejercer funciones en el área de la Prevención.

Los cambios sociales emergentes en los últimos tiempos en América Latina condicionan un análisis de reformas en educación permanente en las áreas de Salud. Tendientes las mismas a renovar las características y mejorar la calidad y eficiencia de los Profesionales. 


\section{INTRODUCCIÓN}

La educación permanente en salud, muy especialmente en el área de la Prevención, compromete a las instituciones formadoras de recursos humanos.

Se buscan nuevos procesos de reforma que mejoren el desempeño profesional, constituyéndose en un desafío continuo de aprendizaje permanente.

La hipótesis de nuestro estudio establece que el área de los contenidos apropiados para una educación permanente se sustenta en una sólida formación pedagógica.

Esta última permite la formación docente para ejercer en el área de prevención.

Actualmente los procesos de formación de recursos humanos en este campo se caracterizan por presentar fenómenos variables según los grados de avance curriculares explorados. 
OBJETIVOS 


\section{OBJETIVOS}

\section{GENERAL}

- Identificar los contenidos conceptuales, procedimentales y actitudinales incluidos en el modelo de Formación continua y permanente, apropiados para formar al Odontólogo en el área de Educación para la Salud

y habilitarlo para ejercer su función en la práctica de la Prevención. 


\section{OBJETIVOS}

\section{ESPECÍFICOS}

- Identificar necesidades prioritarias de formación en Prevención en las universidades.

- Identificar los servicios de Salud para lograr la integración de formación universitaria y demandas sociales.

- Lograr la integración curricular de todos los componentes.

- Examinar los problemas conceptuales y metodológicos generales y las estrategias posibles.

- Formular recomendaciones específicas sobre el currículum de las ciencias de la salud.

- Programar la educación continua. 
DISEÑO METODOLÓGICO 


\section{DISEÑO METODOLÓGICO}

Luego de haber realizado una indagación acerca de la existencia de trabajos previos sobre la temática a abordar, se optó por un estudio de tipo descriptivo, debido a que los pocos antecedentes encontrados, tanto en la Unidad Ejecutora como en otras instituciones similares, abordan la cuestión desde un enfoque en un nivel macro, acerca de los modelos en formación de recursos humanos en salud.

Por lo tanto, se requiere una primer mirada sobre la situación actual de las estrategias de enseñanza en este campo, que permita en una segunda instancia de investigación, intentar avanzar hacia una re-conceptualización de las mismas desde lineamientos didácticos construidos específicamente para estas disciplinas.

Realizamos un estudio de tipo descriptivo con un enfoque cualicuantitativo.

En el siguiente cuadro se expone sintéticamente la matriz de datos con la que se trabajó: 


\section{DISEÑO METODOLÓGICO}

- UNIDAD DE ANÁLISIS

Estrategias de Enseñanza de las especialidades Clínicas en la Carrera de Odontología.

- VARIABLES

Supuestos Epistemológicos:

- Acerca del propio campo disciplinar.

- Acerca del aprendizaje.

- Abordaje metodológico.

- INDICADORES

- Definición del propio objetivo de estudio.

- Organización lógica del contenido.

- Contenidos seleccionados.

- Relación con otras disciplinas (básicas y clínicas).

- Operación cognitivas.

- Tipos de aprendizaje.

- Actividades que se realizan.

- Secuenciación. 


\section{DISEÑO METODOLÓGICO}

La recolección de información se realizó utilizando tanto fuentes primarias, tales como observación de clases y entrevistas a informantes claves, como fuentes secundarias, tales como las programaciones.

El presente trabajo persigue mediante la constatación de la observación de la práctica con la normativa teórica sobre la preparación del odontólogo en el área de salud y prevención explicar y comprender las causas del fracaso práctico, prácticas de la educación recibida en dicha área y elaborar lineamientos para la construcción de modelos didácticos, capaces de responder a la realidad de las prácticas y a las demandas teóricas, metodológicas, disciplinares y a las demandas sociales, vinculadas con las prácticas de actividades de prevención.

Se dio cumplimiento de la expuesto de la siguiente manera: 


\section{DISEÑO METODOLÓGICO}

\section{TRABAJO DE CAMPO}

- Recuperación de prácticos a partir de observaciones en los niveles del sistema educativo en odontología. Las observaciones se complementaron con entrevistas abiertas y semiestructuradas a profesores, alumnos y población en general.

- Búsqueda, selección y clasificación de elementos de la normativa teórica en textos y documentos seleccionados para la posterior constatación con el discurso teórico y las prácticas reveladas.

- Constatación crítica para la explicación e interpretación de las divergencias entre la teoría y la práctica de la prevención y las demandas que la sociedad y las instituciones educativas formulan.

- Elaboración de conclusiones que permitan la construcción de lineamientos de modelos didácticos capaces de orientar el tratamiento de inserción de la prevención en el proyecto educativo general a partir de la detección de los problemas propios de la disciplina. 


\section{DISEÑO METODOLÓGICO}

El estudio se llevó a cabo en la población de profesores, docentes y alumnos de las diferentes asignaturas de la Carrera de Odontología de la Facultad de Odontología de la Universidad Nacional de La Plata. Integrantes de la comunidad de la zona de Melchor Romero que se acercan a los consultorios de extramuros para recibir educación para la salud y esclarecer sus dudas sobre temas relacionados a la salud bucal por parte de docentes coordinadores y alumnos de 10 a 5o año de la Carrera integrantes de la asignatura Odontología Preventiva y Social.

Este trabajo es un testimonio, ya que es producto de una línea de investigación desarrollada dentro de un mismo campo de conocimiento especializado y en contacto directo con los actores, docentes, alumnos y comunidad, con los que se estableció una dedicación sostenida. Se inicio con una fase exploratoria a través de la realización de observaciones, entrevistas en profundidad y encuestas a estudiantes de la Carrera de Odontología. 


\section{DISEÑO METODOLÓGICO}

Se elaboraron informes de base de los temas centrales que pudieron ser indagados. Los datos fueron procesados con Epilnfo 6.0 (ede).

Se analizaron para cada pregunta diferencias significativas entre respuestas (prueba chi cuadrado, $p<0.05)$.

La información recogida permitió caracterizar la transición de la teoría a la práctica.

Se analizaron las encuestas a docentes y comunidad cuyos resultados deducen como indispensable para validar el perfil profesional que la comunidad necesita y quiere tener.

Las encuestas arrojaron datos para impulsar una metodología de educación permanente, como un mecanismo de dinamización y actualización permanente, por lo que decimos que todas las instancias de coordinación son necesarias e imprescindibles para obtener una formación de recursos humanos en salud capaces de responder a las demandas de la sociedad. 


\section{RESULTADOS}

AL LLEVAR A LA PRÁCTICA LAS METODOLOGÍAS PARA RELEVAMIENTO DE DATOS LOS RESULTARON FUERON: 


\section{RESULTADOS}

\section{ENCUESTA A PROFESORES TITULARES Y ADJUNTOS}

Sobre 22 encuestas realizadas a Profesores Titulares y 65 encuestas realizadas a Profesores Adjuntos, con variables relacionadas a las acciones de enseñanza; contenidos del programa; estrategias de enseñanza; formación en roles sociales y calificación del desarrollo profesional en el área de la prevención y la educación permanente; no observamos diferencias significativas entre la mayoría de las respuestas de Profesores Titulares y Adjuntos ( $p>0.05$ ).

\section{ENCUESTA A DOCENTES}

Los resultados obtenidos sobre un total de 39 docentes encuestados nos indicaron que el $85 \%$ posee formación docente, especialización a través de estudios de postgrado; carrera docente. Con respeto a su formación docente, en los aspectos que se vinculan con la prevención de la salud, la mayoría requieren ampliación en esa área de formación, como así también demandan para optimizar su actividad docente, actualización constante. 


\section{ENCUESTA A DOCENTES}

ANÁLISIS EFECTUADO SOBRE 39 ENCUESTAS A LOS DOCENTES 
$15 \%$ NO

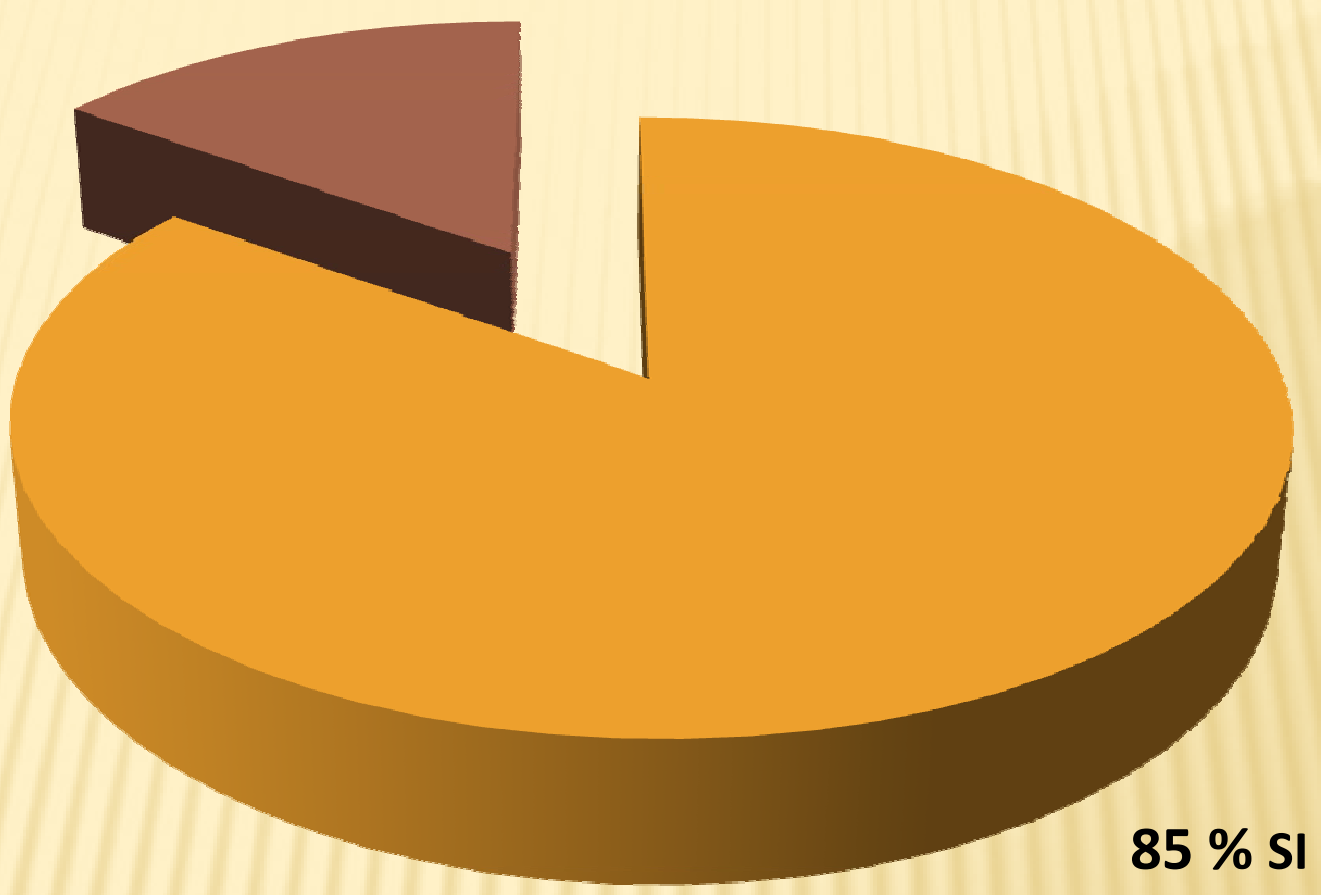

POSEE FORMACIÓN DOCENTE ? 


\section{7 \% ESPECIALIZACIÓN}

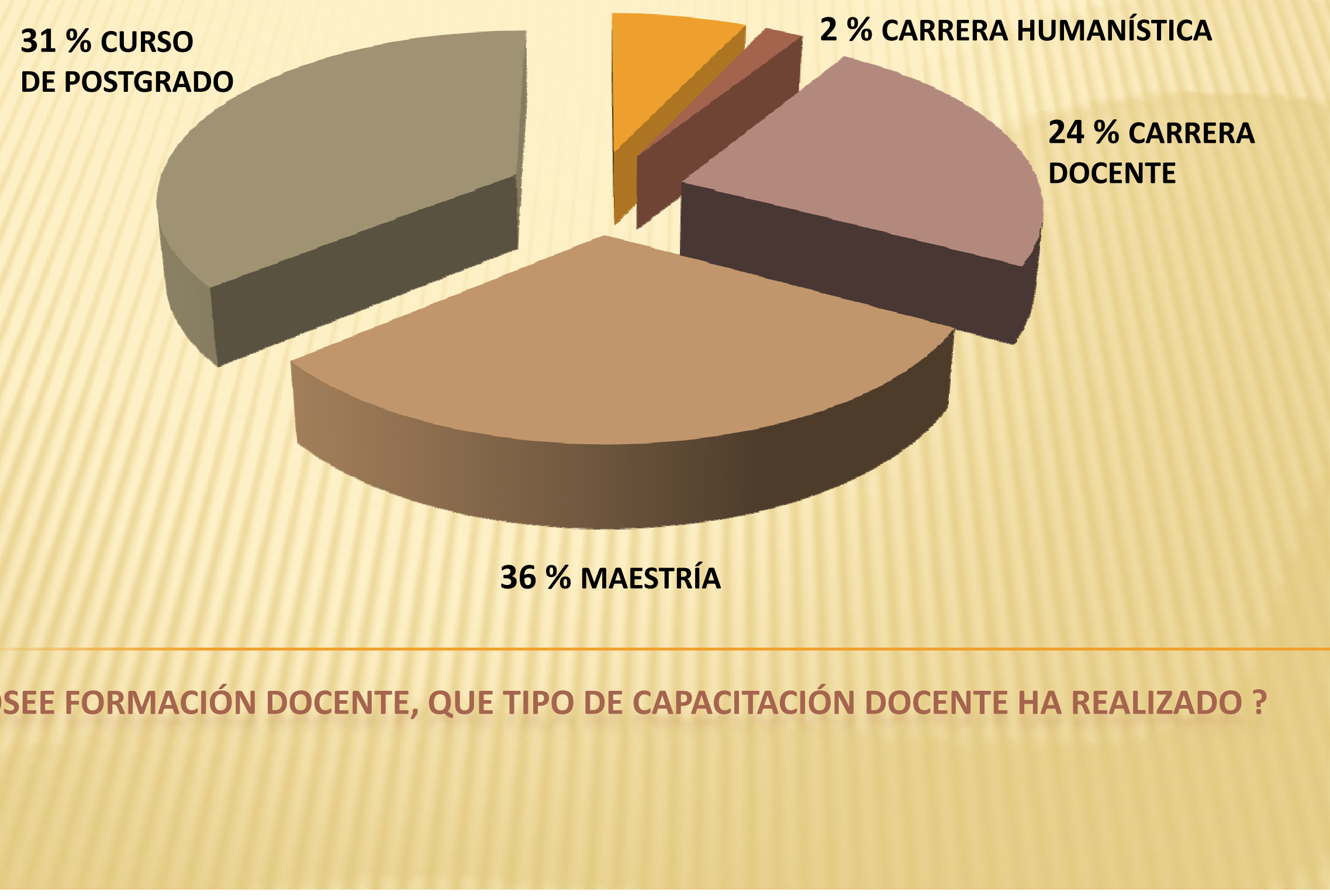


$3 \%$ NS/NC

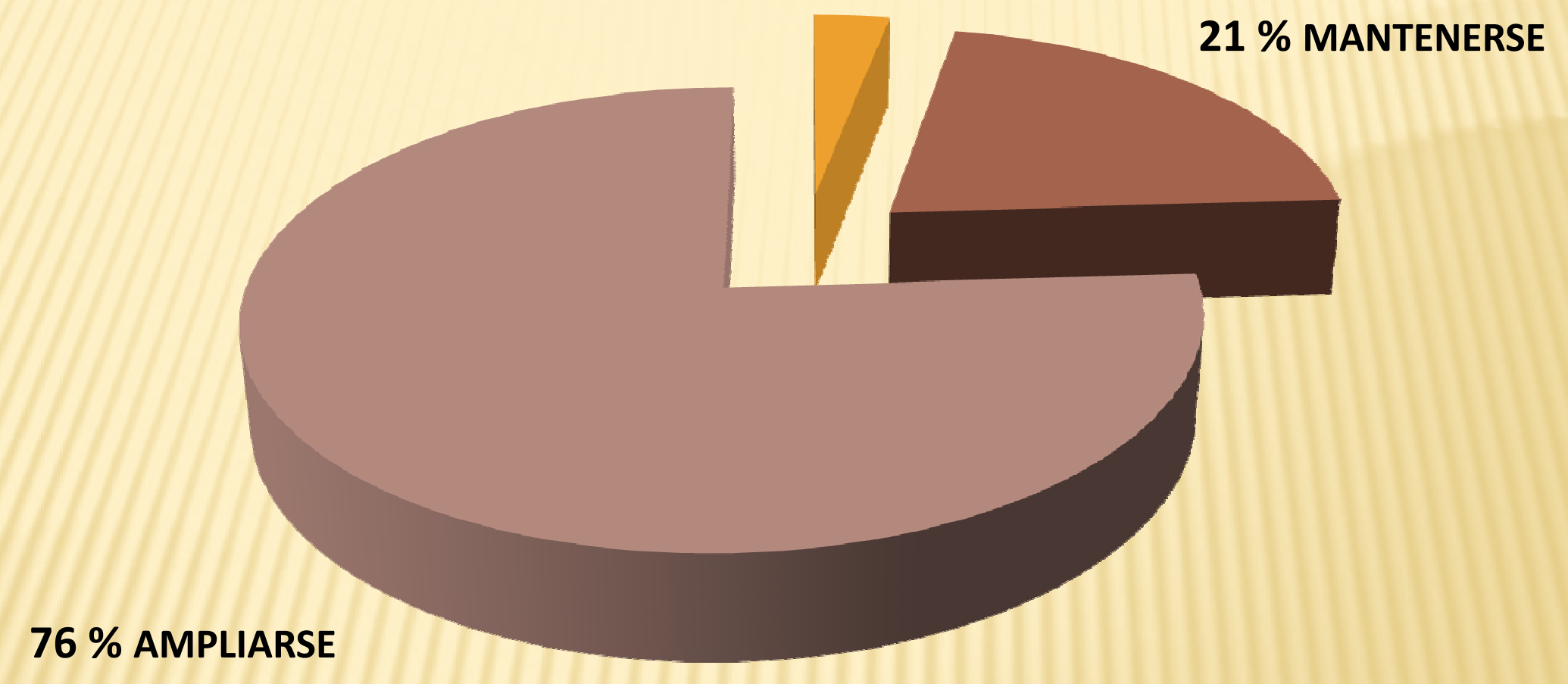

CON RESPECTO A SU FORMACIÓN COMO DOCENTE, EN LOS ASPECTOS SOBRE LA PREVENCIÓN EN SALUD, CONSIDERA QUE ÉSTA DEBERÍA .... 
1 \% RELACIÓN CON LOS ALUMNOS

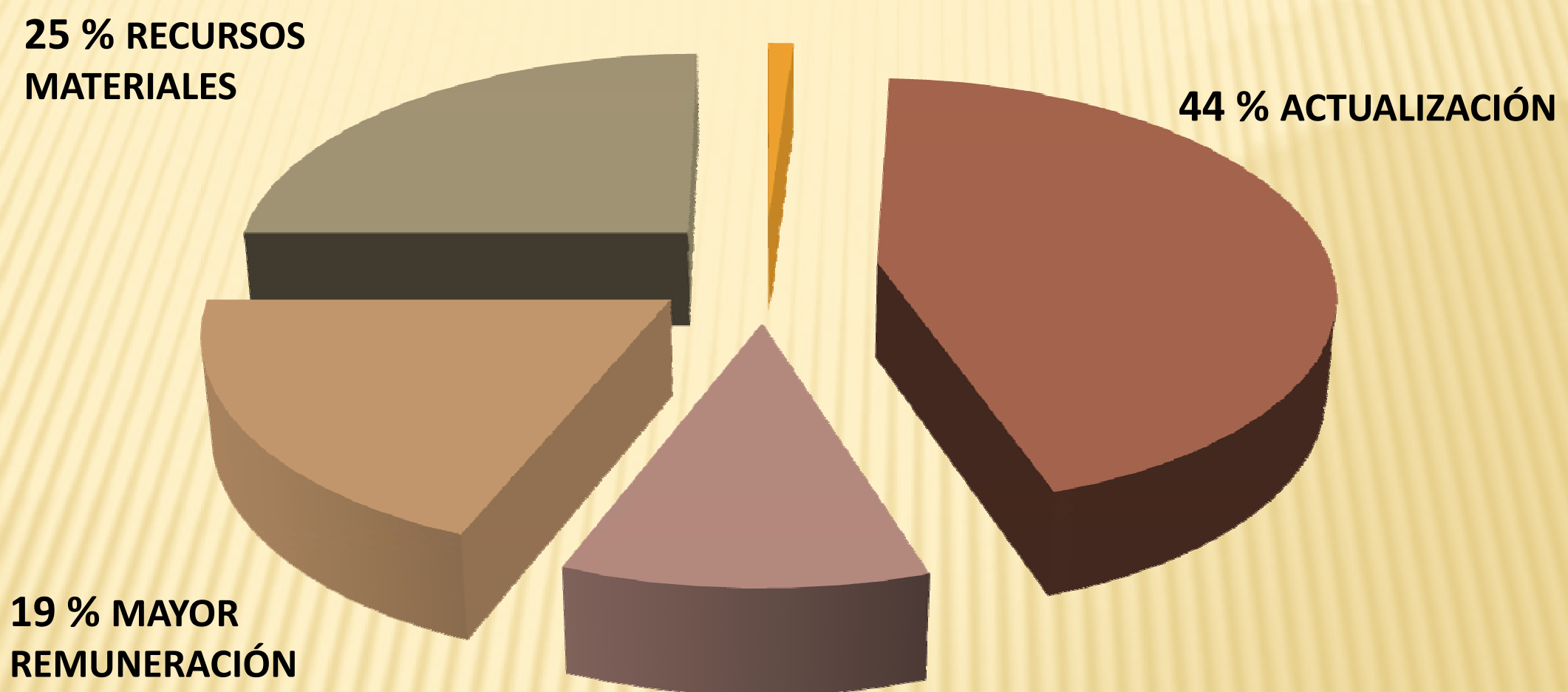

$11 \%$ TIEMPO DE DEDICACIÓN

CUÁLES SON LOS ASPECTOS QUE A SU CRITERIO USTED DEBERIA MEJORAR PARA OPTIMIZAR SU ACTIVIDAD DOCENTE ? 


\section{1ㅇ DIFERENCIA}

INDIQUE SI LA PLANIFICACIÓN DE SUS CLASES TIENE BASAMENTO EN ALGUNA TEORÍA DE ENSEÑANZA/APRENDIZAJE

Con respecto a la planificación de las clases y a la adhesión a alguna teoría de enseñanza/aprendizaje, observamos diferencias significativas entre respuestas de Titulares y Adjuntos $(p<0.05)$.

\section{PROFESORES TITULARES}

Sobre 22 encuestas realizadas a Profesores Titulares

\begin{tabular}{|c|c|}
\hline RESPUESTAS & \% \\
\hline SI & 95,5 \\
\hline NO & 4,5 \\
\hline NS/NC & 0 \\
\hline TOTAL & 100 \\
\hline
\end{tabular}




\section{PROFESORES ADJUNTOS}

Sobre 65 encuestas realizadas a Profesores Adjuntos

\begin{tabular}{|c|c|}
\hline RESPUESTAS & \% \\
\hline SI & 61,5 \\
\hline NO & 32,3 \\
\hline NS/NC & 6,2 \\
\hline TOTAL & 100 \\
\hline
\end{tabular}

\section{CONCLUSIONES}

Se observan diferencias significativas entre respuestas de Titulares y Adjuntos $(p<0.05)$. 
ENCUESTA A PROFESORES 


\section{2o DIFERENCIA}

\section{INDIQUE SI LA FORMACIÓN DEL EGRESADO RESPONDE A LA BÚSQUEDA DE MEJORAMIENTO}

DE LA CALIDAD DE VIDA DE LA POBLACIÓN

En cuanto a si la formación del egresado responde a la búsqueda de mejoramiento de la calidad de vida de la población, encontramos diferencias significativas entre respuestas de Titulares y Adjuntos $(p<0.05)$.

\section{PROFESORES TITULARES}

Sobre 22 encuestas realizadas a Profesores Titulares

\begin{tabular}{|c|c|}
\hline RESPUESTAS & $\%$ \\
\hline TOTALMENTE & 59,1 \\
\hline PARCIALMENTE & 22,7 \\
\hline NS/NC & 9,1 \\
\hline ESCASAMENTE & 4,5 \\
\hline NO RESPONDE & 4,5 \\
\hline TOTALMENTE & 100 \\
\hline
\end{tabular}




\section{PROFESORES ADJUNTOS}

Sobre 65 encuestas realizadas a Profesores Adjuntos

\begin{tabular}{|c|c|}
\hline RESPUESTAS & $\%$ \\
\hline TOTALMENTE & 38,5 \\
\hline PARCIALMENTE & 46,2 \\
\hline NS/NC & 13,8 \\
\hline ESCASAMENTE & 1,5 \\
\hline NO RESPONDE & 0 \\
\hline TOTALMENTE & 100 \\
\hline
\end{tabular}

\section{CONCLUSIONES}

Se observan diferencias significativas entre respuestas de Titulares y Adjuntos $(p<0.05)$. 


\section{3ㅇ DIFERENCIA}

CALIFIQUE EN QUE MEDIDA LOS CONOCIMIENTOS IMPARTIDOS EN SU CÁTEDRA DAN SOLUCIÓN AL EGRESADO PARA LA RESOLUCIÓN DE PROBLEMAS DE LA PRÁCTICA PREVENTIVA

\section{PROFESORES TITULARES}

Sobre 22 encuestas realizadas a Profesores Titulares

\begin{tabular}{|c|c|}
\hline RESPUESTAS & $\%$ \\
\hline AMPLIAMENTE & 86,4 \\
\hline PARCIALMENTE & 9,1 \\
\hline NO DAN SOLUCIÓN & 4,5 \\
\hline ESCASAMENTE & 0 \\
\hline NS/NC & 0 \\
\hline TOTAL & 100 \\
\hline
\end{tabular}




\section{PROFESORES ADJUNTOS}

Sobre 65 encuestas realizadas a Profesores Adjuntos

\begin{tabular}{|c|c|}
\hline RESPUESTAS & $\%$ \\
\hline AMPLIAMENTE & 55,4 \\
\hline PARCIALMENTE & 30,8 \\
\hline NO DAN SOLUCIÓN & 9,2 \\
\hline ESCASAMENTE & 3,1 \\
\hline NS/NC & 1,5 \\
\hline TOTAL & 100 \\
\hline
\end{tabular}

\section{CONCLUSIONES}

Se observan diferencias significativas entre respuestas de Titulares y Adjuntos $(p<0.05)$. 


\section{ENCUESTA A LOS ALUMNOS}

ANÁLISIS EFECTUADO SOBRE 50 ENCUESTAS A LOS ALUMNOS 


\section{$2 \%$ MALA}

$10 \%$ EXCELENTE

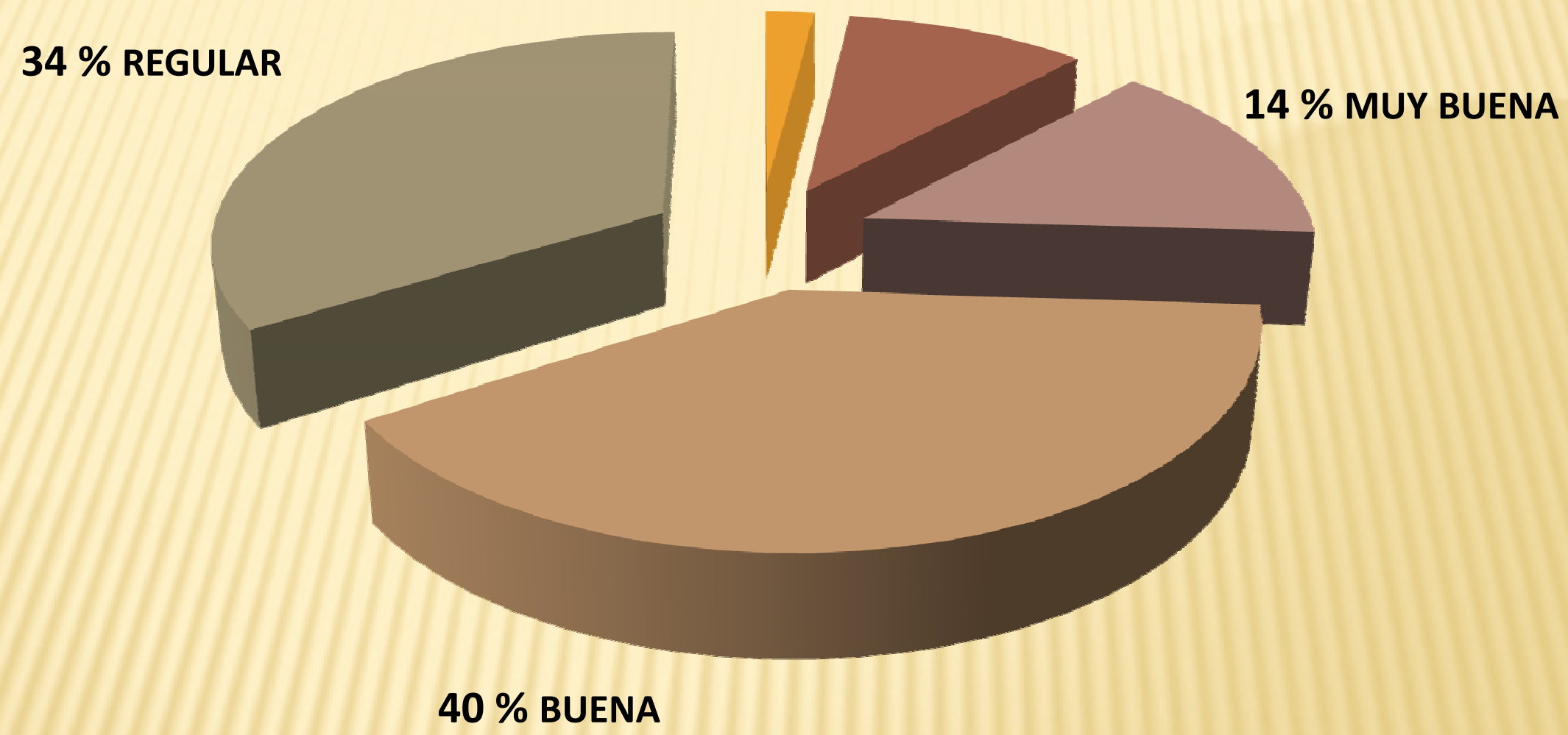

COMO CALIFICARÍA, EN TÉRMINOS GENERALES LA RELACIÓN DOCENTE ALUMNO EN EL TRANSCURSO DE LA CARRERA ? 


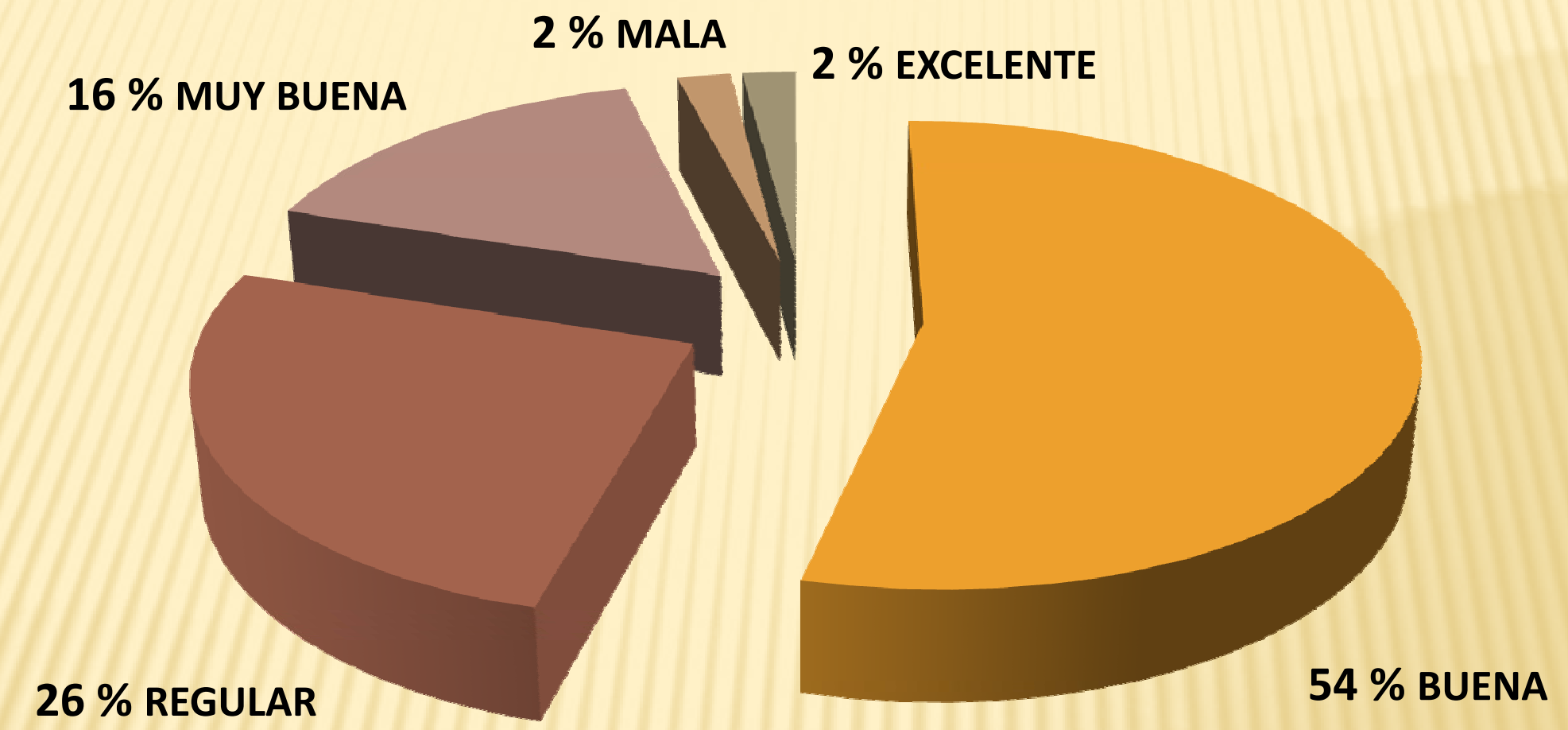

COMO CONSIDERA EL ABORDAJE DE CONTENIDOS QUE TENGAN QUE VER CON LA PREVENCIÓN DE SALUD BUCAL, EN LAS DISTINTAS MATERIAS DE LA CARRERA? 


\section{$32 \%$ NO}

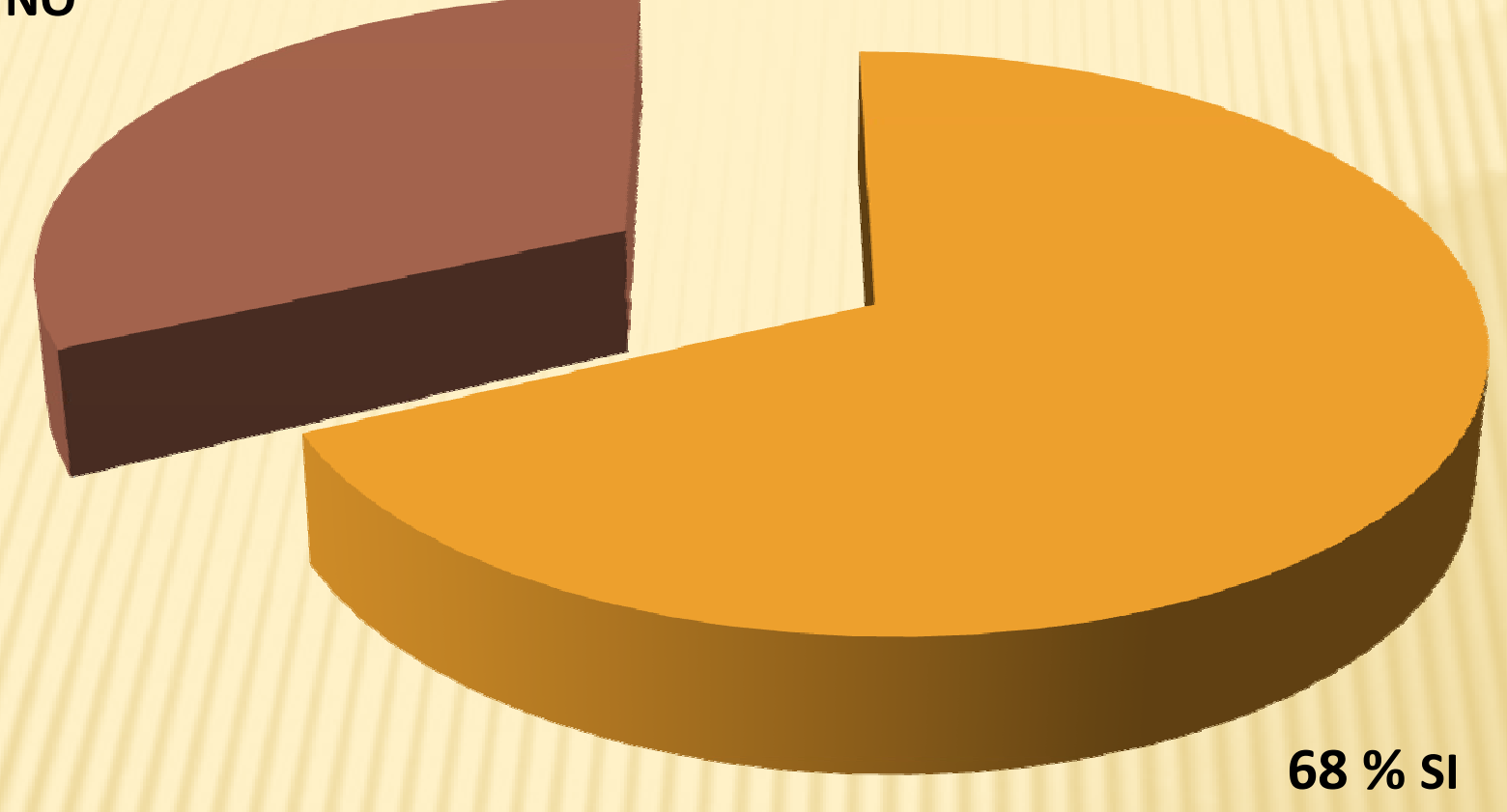

A SU CRITERIO, DEBERIA AMPLIARSE A LO LARGO DE LA CARRERA LA FORMACIÓN DEL ODONTÓLOGO PARA LA PREVENCIÓN ? 


\section{ENCUESTA A LA COMUNIDAD}

ANÁLISIS EFECTUADO SOBRE 41 ENCUESTAS A LA COMUNIDAD 


\section{ENCUESTA A LA COMUNIDAD}

Las encuestas realizadas a 41 representantes de la comunidad de la zona de Melchor Romero arrojaron datos respecto a la formación de los Odontólogos y su relación con ellos.

El 57 \% piensa que la relación Odontólogo/Paciente es buena; que la formación del Odontólogo es buena, referente a las demandas que le harían al Profesional de la salud para el cuidado y la atención de la salud bucal, el $52 \%$ solicita más información sobre prevención. 


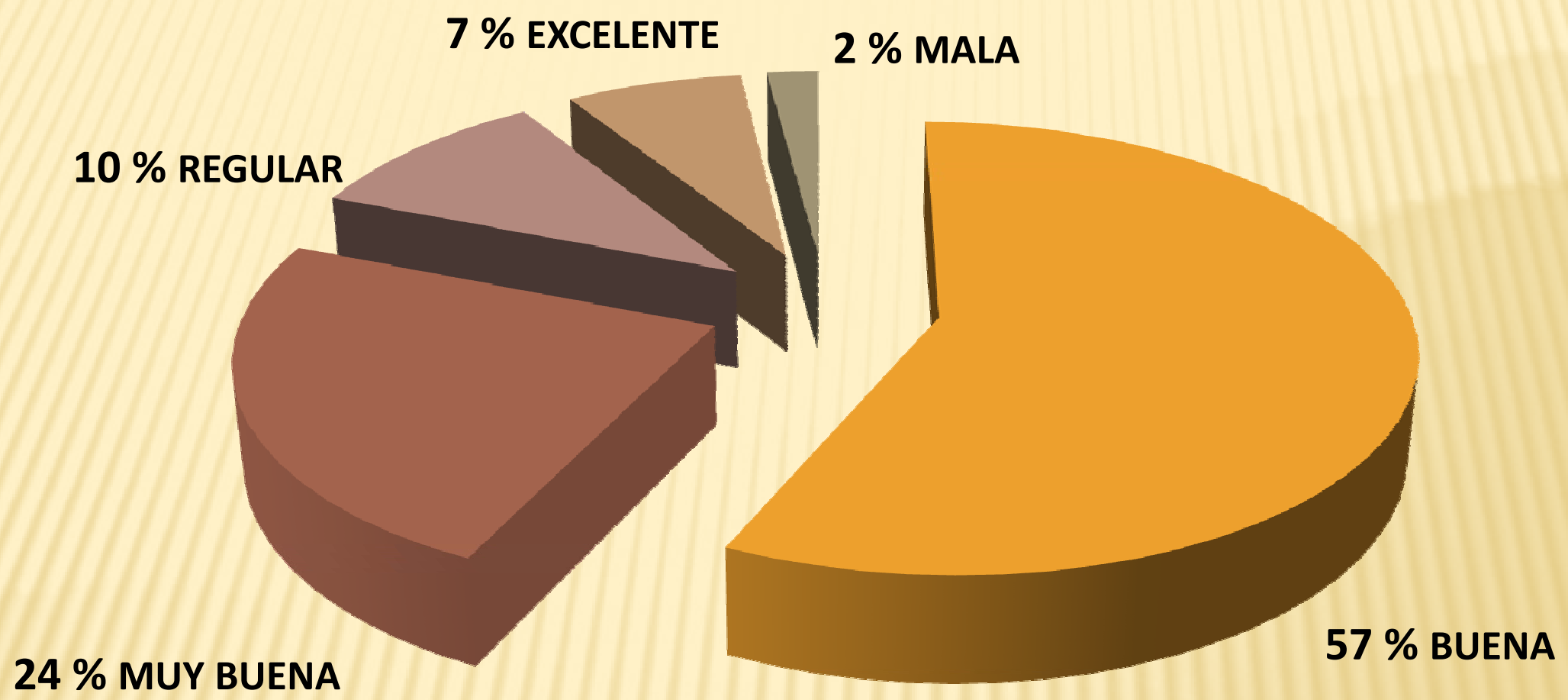

QUE PIENSA ACERCA DE LA FORMACIÓN DEL ODONTÓLOGO, TENIENDO EN CUENTA LOS SIGUIENTES ASPECTOS ? RELACIÓN ODONTÓLOGO/PACIENTE 


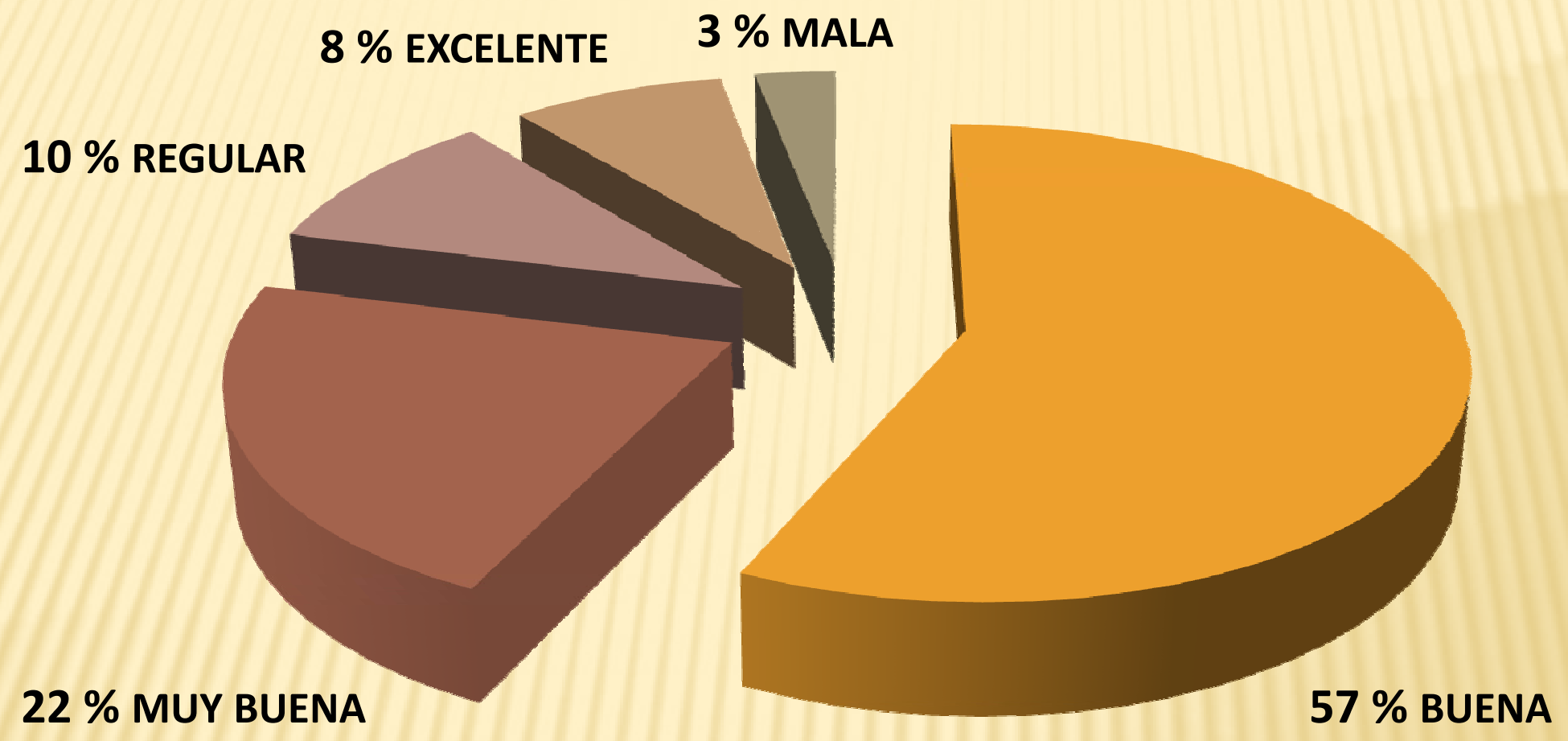

QUE PIENSA ACERCA DE LA FORMACIÓN DEL ODONTÓLOGO, TENIENDO EN CUENTA LOS SIGUIENTES ASPECTOS ? CONOCIMIENTO DEL ODONTÓLOGO SOBRE LAS TAREAS QUE REALIZA EN LA ATENCIÓN DE LA SALUD BUCAL 


\section{$5 \%$ EXCELENTE}

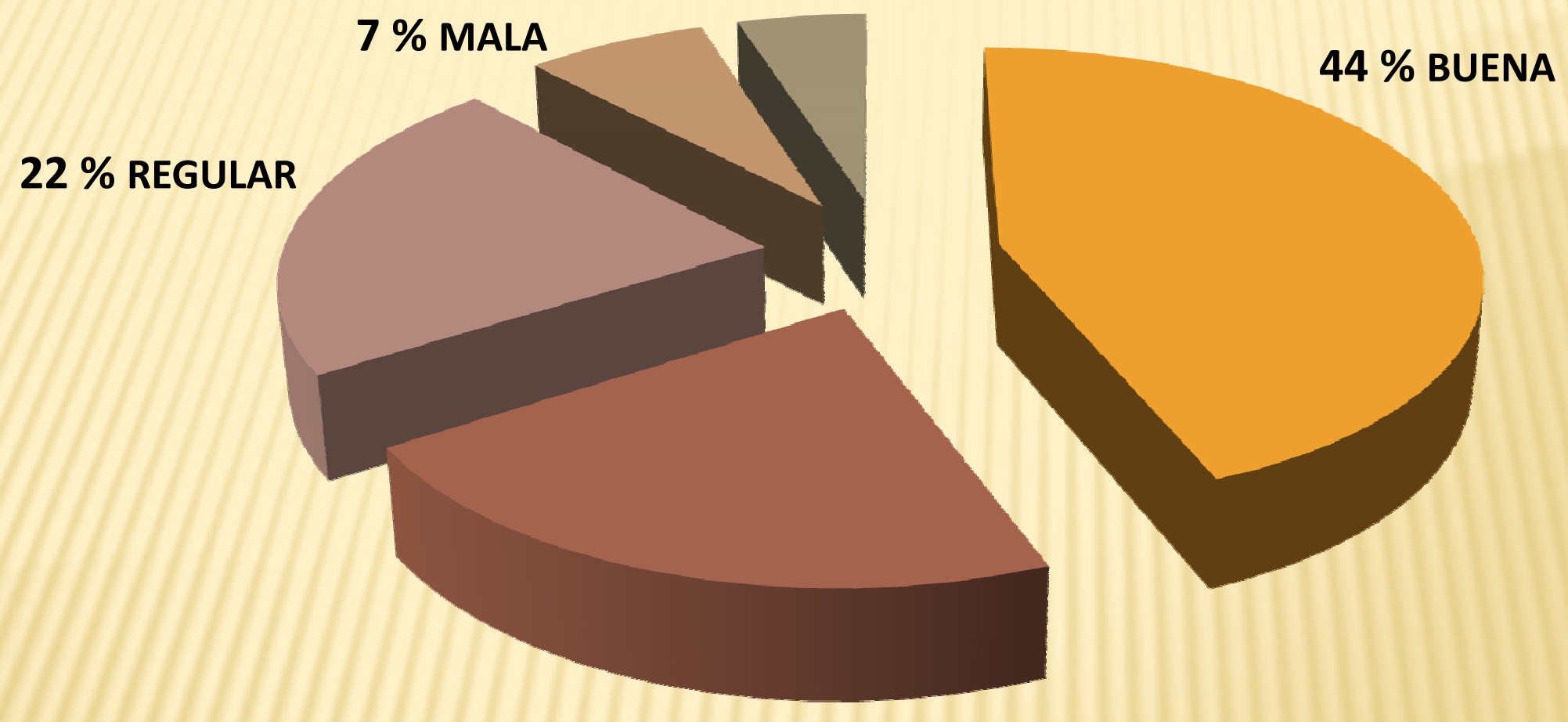

$22 \%$ MUY BUENA

QUE PIENSA ACERCA DE LA FORMACIÓN DEL ODONTÓLOGO, TENIENDO EN CUENTA LOS SIGUIENTES ASPECTOS ? CONTENCIÓN DEL PROFESIONAL HACIA EL PACIENTE CUANDO REALIZA UN DIAGNÓSTICO 


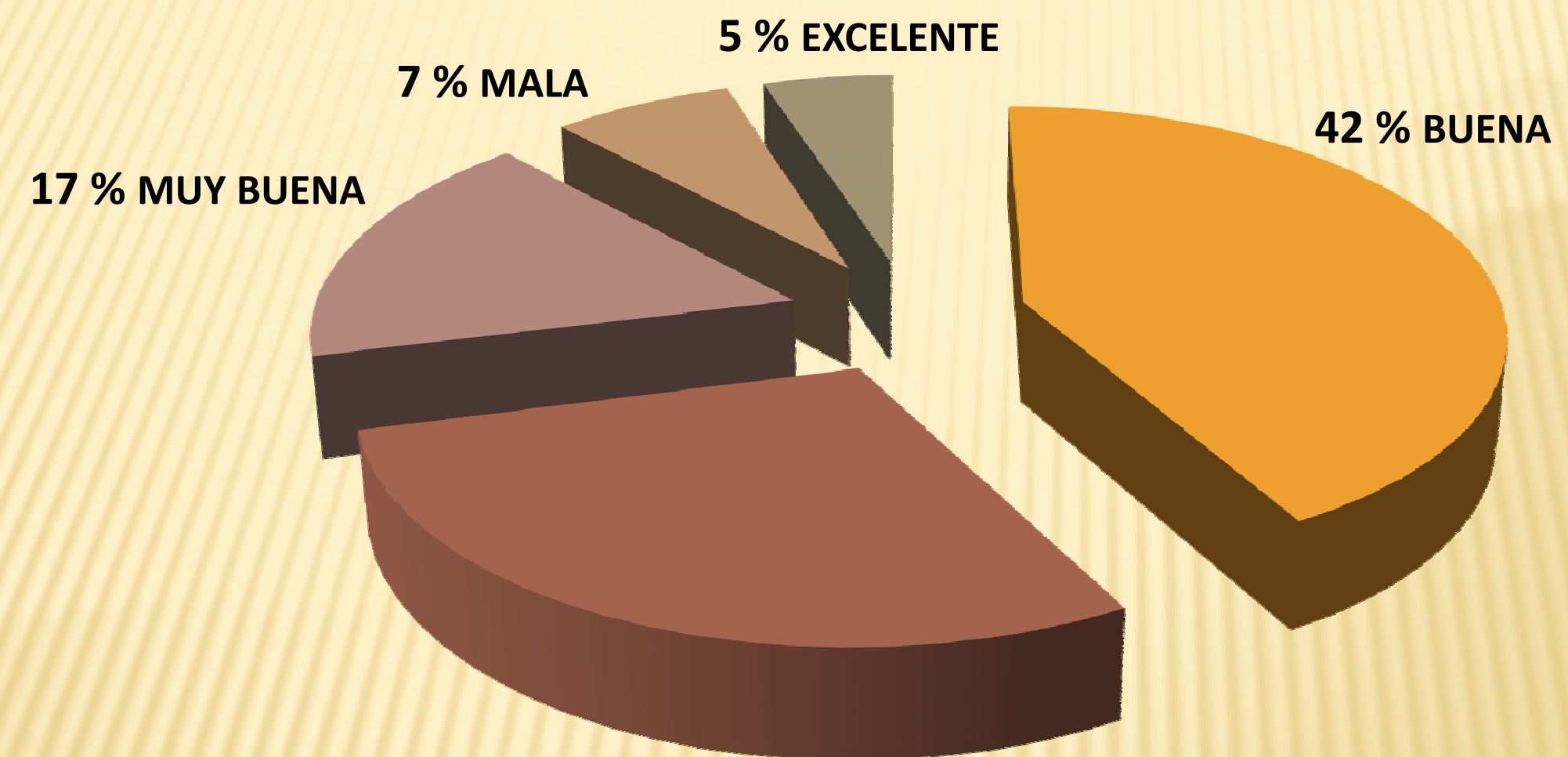

$29 \%$ REGULAR

QUE PIENSA ACERCA DE LA FORMACIÓN DEL ODONTÓLOGO, TENIENDO EN CUENTA LOS SIGUIENTES ASPECTOS? INFORMACIÓN QUE LE DA EL ODONTÓLOGO PARA PREVENIR ENFERMEDADES BUCALES 
OBSERVACIONES DE CLASES 


\section{OBSERVACIONES DE CLASES}

Para analizar la formación de las instancias enseñanza/aprendizaje se realizaron observaciones de clases.

De las 51 observaciones de clases dictadas por Profesores Titulares, Adjuntos, Jefes de Trabajos Prácticos y Auxiliares de Cátedra, se desprende que, si bien los docentes con mayor jerarquía académica (Titulares y Adjuntos) tienen un muy buen manejo de los contenidos específicos de su Asignatura, suele ser escasa la participación activa y motivadora del alumno es un porcentaje de experiencias áulicas.

Hay clases expositivas, tradicionales, con un Docente activo y un Alumno pasivo.

Existe tendencia en los jóvenes docentes auxiliares alumnos buscar una mayor participación del alumno, a través de diferentes estrategias.

Si bien la relación Docente/Alumno, en general, puede ser calificada como buena, el alumno asume muchas veces un rol pasivo, al que por comodidad suele adaptarse. 


\section{DISCUSIÓN}




\section{DISCUSIÓN}

Es fundamental apropiar el concepto de la necesidad de la Educación Permanente en la Formación de los Recursos Humanos en la Salud Bucal.

Davini, define a la Educación Permanente como un Balance necesario.

El especialista mexicano Díaz Barriga, recupera su importancia del papel decisivo de los docentes en la construcción de alternativas permanentes en el campo educativo.

Davini plantea un modelo formativo. El punto es no desarrollar procesos de enseñanza contradictorios con los objetivos que se quieren alcanzar.

La mayor parte de las instituciones formadoras en recursos humanos, al respecto brindan ofertas a través de cursos, jornadas, diversos tipos de reuniones que coadyuvan a mejorar esta instancia. 


\section{DISCUSIÓN}

Román Pérez y Díaz López refiriéndose a las reformas educativas actuales definen como normales los aciertos y errores curriculares, en el marco de un nuevo paradigma emergente en el que afloran ideas y prácticas nuevas que se mezclan con prácticas viejas que resultan difíciles de entender y aplicar.

La Comunidad Educativa piensa que el espacio pertinente al área disciplinar de la prevención es adecuado.

Expresa la necesidad de ampliar metodologías, instrumentos, estrategias, para favorecer la interacción y participación activa de la Comunidad en la construcción de planes y proyectos en prevención de la Salud Bucal. 


\section{DISCUSIÓN}

En este estudio hemos hallado diferencias significativas en lo que respeta a la formación del egresado, tales como carencias de actividades y falta de programas que impulsen la educación continua en las instituciones, donde se encuentran muy dispersas y discontinuas, mientras los estudiantes piden mayor tiempo en prácticas preventivas.

La mayor parte de los Docentes poseen alguna especialización, pocos han realizado Maestrías o Carreras específicas.

Rovere manifiesta que pensar en salud es un recorte más o menos artificial de la realidad social en donde se desarrolla y constituye el proceso salud-enfermedad como un objeto-sujeto social.

Los encuestados de nuestra investigación sugieren una constante actualización. 


\section{DISCUSIÓN}

Raschke Brito Quintana, Palacios, Davini y Rovere describen procesos de reforma articulados con el medio social, creando un nuevo escenario para la educación permanente que involucran nuevos desafíos y desempeños en la formación de recursos humanos en salud.

Consideramos importante la intervención de la comunidad, esta investigación reveló distintos aspectos (relación odontólogo-paciente-atención-contención-información), la comunidad los consideró como buenos, sin lugar a dudas surgirán demandas respecto a información, relación, disponibilidad de profesionales; entre otras cuestiones.

Acá cabe puntualizar el rol de las instituciones formadoras. Foucault distingue el rol constituyente que las instituciones tienen sobre la sociedad.

Existen incongruencias respecto a la práctica; hábitos no válidos; tasa de olvido, cambios tecnológicos, sociales, epidemiológicos, ambientales, etc., lo que nos conduce a repensar el rol de la Educación permanente en la formación docente en todos los ámbitos y especialmente en la prevención. 


\section{DISCUSIÓN}

Haddad Raschke y Casas opinan sobre un eje de integración entre la teoría y la práctica para dar una respuesta a la dinámica política, social y epidemiológica de determinada realidad. Los recursos humanos en odontología, constituyen un eje central para dar lugar a esa respuesta.

Los entrevistados de nuestro estudio, graduados y estudiantes, sostienen que los cambios curriculares realizados para formar al profesional en la Prevención y Preservación de la Salud son adecuados.

Al decir de Davini, es consenso que la Educación es un proceso continuo que acompaña y atraviesa todo la vida del hombre.

Mejía, concibe la Educación continua como "conjunto de experiencias que sigue a la formación inicial y que permite al trabajador mantener, aumentar y mejorar su competencia para que ésta sea pertinente al desarrollo de sus responsabilidades". "La Educación inicial no garantiza un ejercicio profesional idóneo indefinidamente, aporta los conocimientos para continuar la Educación durante toda la vida profesional". 


\section{DISCUSIÓN}

Deberían ampliarse estrategias didácticas que permitan orientar hacia una Educación permanente en Salud y Prevención.

Los Estudiantes y el Egresado deben concientizarse de un compromiso profesional y social con las metas de Salud.

Respecto a la ampliación de las estrategias didácticas, pretendemos un docente que oriente y estimule, que el Estudiante pueda descubrir, a partir de la reflexión y elaboración, el saber. En este punto resultan interesantes los interrogantes de Davini a través de la "pedagogía de la pregunta" ¿cómo hago?, ¿qué dificultad encuentro, cuando lo hago?, ¿en qué situación lo hago?, ¿Por qué lo hago de esta manera?.

La mejor manera de resolver estas cuestiones es el "pensar en grupo".

Para este efecto, se debe proporcionar en las instituciones formadoras en ámbito participativo, buena integración docente/asistencial y sólidos principios pedagógicos. Por otro lado, la programación debe darle un lugar específico a conocimientos de prevención en Salud oral. 


\section{CONCLUSIÓN}

El análisis de Educación permanente en el área de la Prevención, en la formación docente en Odontología, constituye un requisito ineludible. Sin embargo, no representa un campo de investigación muy desarrollado.

Nosotros consideramos muy importante la capacitación permanente en Prevención.

Los cambios curriculares tienden a mejorar la formación de los Alumnos en Prevención, pero opinamos que la acción impartida por los Docentes debe ser compartida y acordada por las diferentes asignaturas que componen la Carrera y no restringirse a unas pocas. 


\section{CONCLUSIÓN}

Los tiempos actuales requieren programas de Educación permanente en Salud, por lo tanto Recursos Humanos preparados para:

- SOLUCIONAR LOS PROBLEMAS EN SALUD BUCAL DE LA POBLACIÓN

- FORMAR FORMADORES

Se requiere un desarrollo de Recursos Humanos en Salud con una formación metodológica sin la cual se reducen las posibilidades de intervención eficaz. 


\section{CONCLUSIÓN}

El sistema de salud actual debe encarar cambios, para enfrentar de manera adecuada los problemas de Salud. Se necesitan acciones conjuntas, un eslabón de ese conjunto lo constituyen las Instituciones formadoras de recursos humanos en Odontología, específicamente en el área de la Prevención, se debe tender a que los egresados sean capaces de operativizar nuevas y eficaces estrategias para prevenir la aparición de enfermedades.

Según la data recabada y nuestras experiencias, un modelo de formación continua en Prevención debe ser realista y factible con apoyo institucional, material, financiero del contexto, de los formadores, con objetivos claros de formación.

La Educación permanente en estos tiempos se transforma en un instrumento estratégico para enfrentar cambios a corto y largo plazo en Salud. 
" Uln ansia infinita de pas, un esfuerzo de superación el amor al bien $y$ el mejoramiento social de las personas " 